\title{
The Universe is Almost All Perfect, Accurate, Regular and Appropriate but Never All Perfect or Accurate or Regular or Appropriate: The Universe Only Has The Knife Edge Accuracy or Large Scale Accuracy but not Absolute Accuracy
}

\author{
Prasenjit Debnath
}

PhD Student, NIT Agartala, India

\begin{abstract}
On the large scale of the Universe, everything in the Universe is so fine tuned for the evolution of living being on Earth. We can term the fine tuned Universe as the Universe at the knife edge accuracy or mere the large scale accuracy. But the Universe is not at the absolute accuracy, for that, the necessary condition is that the Universe must be totally evenly distributed in all sides in both macroscopic and microscopic scale. There are variations in distribution of matters in microscopic scale, although on large scale it is almost all perfect, accurate, regular and appropriate. The almost word seeks attention due to variations (at times huge variations within a local area) in the microscopic scale. There is a sense of irregularity in the very regular and well behaved Universe - the gentle Universe on a large scale. On psychological point of view, the Universe is too gentle (sometimes more than necessity psychologically) because to take place cosmic changes of evident, it takes minimum of million years if not billion years. We pass our life by seeing the Universe almost unchanged. Nature hates sharp changes and nature loves to hide everything. Because human beings are confined by nature in a small area for a small amount of time, we are basically microscopic structure of the Universe; as a result, small scale changes in local area nearby are evident to us, not the large scale changes or cosmic changes. While 70 years is everything for us, for Earth, it is mere tiny little time seeks no attention. Even the Earth is so huge for us, 200 years of technological revolution hurt the Earth that much that the average temperature of the Earth increased by 0.85 degree centigrade which we call global warming due to release of green house gases; a huge technological boom of human being is easily damped out by the Earth. On a little bigger scale of space, 200 years made nothing changed to Sun or in the solar system, it is almost as it is on a large scale. For example, sun burned almost nothing fuel in 200 years and the orbits of planets have almost nothing change in 200 years - the comfortable time for these changes to be noticeable are million or billion years. The cosmic microwave background (CMB) radiation is nothing but the afterglow radiation left over from the very hot Big Bang. Its temperature is extremely uniform all over the sky. However, tiny temperature variations or fluctuations (at one part of the million level) can be detected which offer great insight into the origin, evolution, and content of the universe - a fluctuation in the CMB tells us a sense of irregularity in the highly uniformed early Universe during the Big Bang. This sense of irregularity is the key for evolution of the Universe of the present state. This very tiny irregularity is carry forward to make the present state Universe as irregular in the microscopic scale and highly regular in the macroscopic scale. Thus it is almost regular but not absolute regular. If the space would be evenly distributed, there would not be formation of energy and mass contents. A sense of irregularity in the distribution of space points developed mass and energy. Thus the Universe is nearly accurate but not absolute accurate because almost all the space remains as free space except very rarely somewhere little deformation made masses and energy content of the Universe. Again mass and energy has uneven distribution with $27 \%$ total mass content and $73 \%$ energy content. This variation is not significant because insignificant portion of space deformed into mass and energy, but still it is irregularity on a microscopic scale of the Universe with respect to free space of reference frame.
\end{abstract}

Keywords: The cosmic microwave background (CMB) radiation, the large scale accuracy, the absolute accuracy, very very regular and well behaved Universe, the free space of reference frame.

\section{Introduction and Theory}

The deformation in the distribution of space-points forms the mass. If the elemental structure of mass is an atom [1,2]there is uneven distribution of space points within the atom. The space points highly squeezed in the nucleus to have most of the mass in the nucleus in terms of protons and neutrons. While most of the space points are unaltered to form the vacant place within the atom but slight deformation of very little space-points forms electrons. Electrons are revolving around the nucleus [3, 4] means the deformed space-points changes position in space with a certain rate can be called electron velocity within the atom. It is like a wave of deformed space-points and the energy that drives the deformed space-points which is velocity of electrons involves time into it. Thus the velocity of electron is nothing but the combinational effect of discrete space points and the associated unit time vector with the discrete space points. The space points can be regarded as elemental representation of space $[5,6]$. The structure of the atom reveals that most of the space points are unaltered within the atom [7, 8], very few highly deformed to form nucleus and some very very less number to form electrons revolving around it $[9,10]$. In that sense, it is a sense of slight irregularity to form space points into mass. Energy that drives the mass with velocity and acceleration [11, 12] also deformed space with active participation of time associated with each space point. A unit time vector can be assumed to be associated with each space point because velocity and acceleration of mass is a function of time $[13,14]$. Thus mass and energy both are function of space and time $[15,16]$ and mass and energy are evolutional product of space and time too $[17,18]$. 


\section{Why the Universe is Almost Accurate but Not Absolute Accurate}

The Universe looks enormously even from every possible sides $[19,20]$, thus it tremendously uniform on a large scale [21] but it is not all true for microscopic structure of the Universe [22]. There is tremendous regularity in the distribution of masses from every side [23], but it is true only for macroscopic scale only, not valid for microscopic scale [24]. The universe follows tremendous accuracy, like for example, if we fall a ball billion times on earth, every time it will be a parabola [25]. If we observe million times the circular path of the Earth around Sun, every time it will be an ellipse. No matter how many times we do the experiment, but we are never sure, next time the result will be the same [26]. We walk with our foot billion times in life, still we accident at least twice or thrice if not even more while we make steps forward, nothing is full proof, thus it is a safe side to tell, the Universe is almost all accurate but not absolute accurate. If the Universe would be absolute accurate, we might not have mass or energy or different times at different places, rather it would be all same space and time everywhere, which can be termed as universal space and universal time. There would not arise question of microscopic or macroscopic structure because, there would not be any structure at all as such now [27, 28]. If mass would not be there, neither Sun nor Earth nor human would be there to ask very intelligent questions - why are we here? Only slightest of sense of irregularity during Baby Universe made it feasible to have the Solar system, the Earth and Human to be as they are now. The slightest of irregularity made the mass and energy and we are intelligent because both mass and energy is working properly as combination in the most complex system of human body [29, 30].

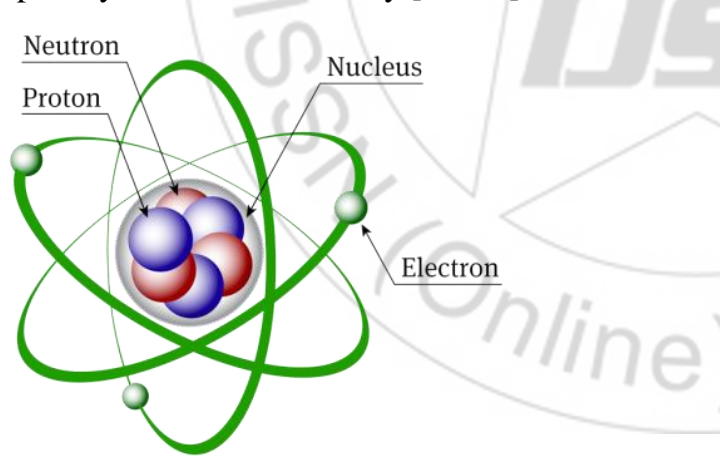

Figure 1: Most of the space of atom is vacant which means that most of the space points are free space points of reference.

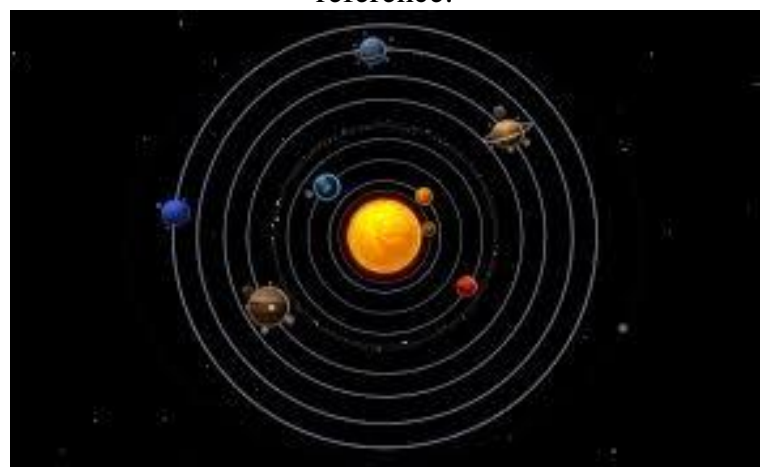

Figure 2: Most of the space of solar system is vacant which means that most of the space points are free space points of reference which resembles an atom

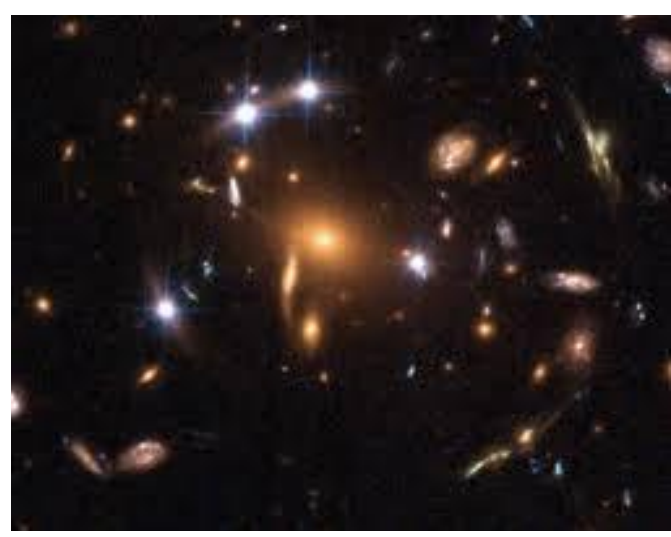

Figure 3: Most of the space of in a cluster of galaxy is vacant which means that most of the space points are free space points of reference which resembles an atom and solar system on a large scale.

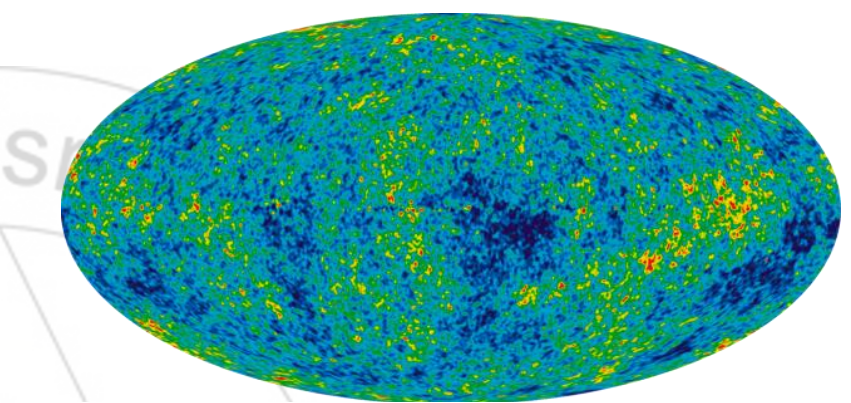

Figure 4: The detailed, all-sky picture of the infant universe created from nine years of WMAP data. The image reveals 13.77 billion year old temperature fluctuations (shown as color differences) that correspond to the seeds that grew to become the galaxies. The color difference reveals slightest of sense of irregularity of the Universe.

Because almost all the space is a free space of reference, with little deformation of space on an atomic scale (fig 1), on solar system (fig 2), on a cluster of galaxies (fig 3), on the observable Universe (fig 4), we can conclude that the Universe is almost accurate, but not absolute accurate. The tiny little variation made the Universe as we look it now.

\section{Conclusion}

The slightest of impurity of doping in the semiconductor with pentavalent atoms or trivalent atoms made drastic change in the electrical property of the semiconductor. At times it looks huge change in the characteristic with slightest of irregularity. The similar way a slightest of irregularity in the early Universe, created mass and energy, the shape of the atom, the solar system, the galaxy clusters and the whole Universe as we see it now. We are concerned about the masses and energies of the Universe which means that we are concerned about the slightest of irregularity of the Universe and not the Universe as a whole (including the free space into it), although almost all the space points of the Universe are free space points of reference. Similarly, we are concerned about the nucleus and electrons of an atom and not the vacant area of the free space inside the atom. Why we are only concerned about the slightest of irregularity only, not as a whole system, astrophysics and cosmology seeks proper explanation of that with a wild guess that it might be another limitation of psychology. On psychological 


\section{International Journal of Science and Research (IJSR) \\ ISSN (Online): 2319-7064}

Index Copernicus Value (2013): 6.14 | Impact Factor (2015): 6.391

ground, drastic changes catches mind, like masses and energies do but insignificant changes ignored by human mind such as free space of reference although masses and energies are insignificant compared to free spaces in the Universe. The slightest of irregularity made us to conclude that the Universe is almost all perfect, accurate, regular and appropriate but never all perfect or accurate or regular or appropriate.

\section{Acknowledgment}

I cordially admire Dr. Aparna Nath, Associate Professor and my $\mathrm{PhD}$ Guide, The department of Physics, National Institute of Technology, Agartala, India, for the epitome of inspiration and motivation to write this particular paper with perfection and accuracy. I am extremely thankful to her from all possible help she made to write this paper. Also I am thankful to The Department of Physics of National Institute Of Technology Agartala (NIT Agartala) for proper conduct and coordination.

\section{References}

[1] http://wmap.gsfc.nasa.gov/universe/bb_cosmo_fluct.ht $\mathrm{ml}$

[2] Lee Smolin, “ Time Reborn: From the Crisis in Physics to the Future of the Universe" Penguin Books, pp. 1-41

[3] Prasenjit Debnath, "Time and Space Are Mutually Inherent Property of Each Other, Where the Dependency of Time on Space Can Be Termed as Space-Time and the Dependency of Space on Time Can Be Termed as Time-Space" International Journal Of Science And Research, ISSN (online)- 2319-7064, volume 5 Issue 2, pp 1946-1948

[4] Brian Cox, Jeff Forshaw, "The quantum universe: everything that can happen does happen", Penguin Books, pp. 1-44.

[5] Stephen Hawking, "A Briefer History of Time", Bantam Books, London, pp. 1-145.

[6] http://map.gsfc.nasa.gov/universe/uni_fate.html

[7] Stephen Hawking, "Black holes and Baby Universes and other essays", Bantam Press, London 2013, ISBN 978-0-553-40663-4

[8] Stephen Hawking, "The Grand Design", Bantam Books, London 2011

[9] Stephen Hawking, "A Brief History of Time", Bantam Books, London 2011, pp. 156-157. ISBN-978-0-55310953-5

[10] Stephen Hawking, "The Universe in a Nutshell", Bantam Press, London 2013, pp. 58-61, 63, 82-85, 90-94, 99, 196. ISBN 0-553-80202-X

[11] Stephen Hawking, "The Beginning of Time", A Lecture.

[12] Stephen Hawking, “Stephen Hawking's Universe: Strange Stuff Explained", PBS site on imaginary time.

[13] Stephen Hawking, "How to build a time machine", 27 April, 2010.

[14] Uno Ingard, K "Fundamental of Waves \& oscillations", Cambridge University Press. P. 38, ISBN-0-521-33957-XOxford: The British Academy, 1999

[15] A. Zee, "Quantum Field Theory in a Nutshell", Princeton University Press, 2003
[16] Storrs McCall, "A Model of the Universe”, Oxford: Clarendon Press, 1994

[17] Craig Callender, "Time, Reality and Experience", Cambridge, UK: Cambridge University Press.

[18] Craig Callender, "Thermodynamic Asymmetry in Time", The Stanford Encyclopedia of Philosophy (Spring 2002 Edition)

[19] Storrs McCall, "A Model of the Universe", Oxford: Clarendon Press, 1994

[20] Robin Le Poidevin and Murray McBeath, "The Philosophy of Time" Oxford: Oxford University Press, 1993

[21] Newton-Smith, W.H., "The Structure of Time". London: Routledge \& Kegan Paul, 1980.

[22] Barry Dainton,"Time and Space", Ithaca: McGillQueen's University Press, 2001

[23] Robin Le Poidevin, "Questions of Time and Tense", Oxford: Oxford University Press, 1998.

[24] Nerhlich, Graham, "What Spacetime Explains". Cambridge: Cambridge University Press, 1994.

[25] Whitrow, G., "The Natural Philosophy of Time". Oxford: Oxford University Press, 1961. (2nd edn., 1980.)

[26] S.W. Hawking, and G.F.R. Ellis, "The Large Scale Structure of Space-Time", Cambridge University Press, (1973).

[27] Stephen Hawking, "A stubbornly persistent illusionThe essential scientific works of Albert Einstein", Running Press Book Publishers, Philadelphia, London 2011.

[28] Flynn, John L, "Time travel literature", on 29-09-2006

[29] Stephen Hawking, "The Theory of Everything", Jaico Books, pp. 1-110.

[30] Stephen Hawking, "The Illustrated A Brief History of Time”, Bantam Books, pp. 1-21.

\section{Author Profile}

Prasenjit Debnath, born in Agartala, Tripura, India on $15^{\text {th }}$ of March 1979. He is pursuing $\mathrm{PhD}$ in Department of Physics in National Institute of Technology Agartala (NIT Agartala), India 